\title{
Investigation of Neuroprotective and Therapeutic Effects of Hesperidin in Experimental Spinal Cord Injury
}

\author{
Ziya YURTAL ${ }^{1}$, Muhammed Enes ALTUG ${ }^{1}$, Emine UNSALDI ${ }^{2}$, Ilke Evrim SECINTI ${ }^{3}$, Altug KUCUKGUL ${ }^{4}$ \\ ${ }^{1}$ Hatay Mustafa Kemal University Faculty of Veterinary Medicine, Department of Surgery, Hatay, Turkey \\ ${ }^{2}$ Firat University Faculty of Veterinary Medicine, Department of Surgery, Elazig, Turkey \\ ${ }^{3}$ Hatay Mustafa Kemal University Faculty of Medicine, Department of Pathology, Hatay-Turkey \\ ${ }^{4}$ Hatay Mustafa Kemal University Faculty of Veterinary Medicine, Department of Biochemistry, Hatay, Turkey \\ This study has been presented at the $1^{\text {st }}$ International Veterinary Biochemistry and Clinical Biochemistry Congress at 18 April 2018 , Hatay, Turkey
}

Corresponding author: Ziya YURTAL ziyayurtal@gmail.com

\section{ABSTRACT}

AIM: To investigate the neuroprotective and therapeutic efficacy of hesperidin against secondary damage following traumatic spinal cord injury.

MATERIAL and METHODS: A total of 32 male Wistar albino rats weighing 250-300 g were randomly divided into four groups ( $\mathrm{n}=4$ ): group I, control group; group II, sham group; group III, preconditioning group, and group IV, treatment group. A rat model of spinal cord injury was established by dropping a weight of $100 \mathrm{~g} / \mathrm{cm}$ on the spinal cord exposed at T7-T10 with dorsal laminectomy. In neurological examination after the trial period, inclined planed test, modified Tarlov scale, and finger extension test were performed. Furthermore, the bioefficacy of hesperidin was investigated histopathologically, biochemically, and immunohistochemically using blood and tissue samples obtained from the experimental animals.

RESULTS: Neurological examination following spinal cord injury revealed that hesperidin significantly contributed to improvement in the 24-hour period. Biochemical analyses revealed that hesperidin showed anti-inflammatory effects by decreasing IL-1 $\beta$ and TNF-a levels at the 24th hour as well as strong antioxidant activity by increasing TAS levels in groups III and IV. Histopathologically, hesperidin reduced hemorrhage, laceration, axonal and neuronal degeneration, necrosis, inflammatory reaction, and edema in groups III and IV. Immunohistochemically, hesperidin reduced the number of caspase 3-positive apoptotic cells in groups III and IV. CONCLUSION: Hesperidin showed antioxidant, anti-inflammatory, and anti-apoptotic effects during the acute period following spinal cord injury; thus, hesperidin shows neuroprotective and therapeutic efficacy in spinal cord injury.

KEYWORDS: Spinal cord injury, Hesperidin, Oxidative stress, Apoptosis, Caspase-3, Rats

\section{INTRODUCTION}

0 pinal cord injuries are very important physical, psychosocial, and economic problems $(9,14,18,34)$. The annual incidence of traumatic spinal cord injury varies between approximately 12.1 and 57.8 per million (20). Despite advances in current surgical techniques, recovery in cases with spinal cord injury remains generally incomplete (14). Furthermore, there are no proven therapeutic agents for curing this complex condition (12).
Spinal cord injury occurs through two mechanisms: primary and secondary. Primary injury refers to the mechanical damage and secondary injury refers to the progressive cell damage after trauma $(34,38)$. After primary injury, hypoxia occurs in the spinal cord followed by reactions such as electrolyte imbalance, neuronal excitation, glutamate release, and inflammation $(5,9)$. Free oxygen radicals, lipid peroxidation, and apoptosis play important roles in secondary injury development in spinal cord injuries $(14,16)$.
Ziya YURTAL

(1) : 0000-0001-6080-1860

Muhammed Enes ALTUG (D): 0000-0003-3896-9944

Emine UNSALDI
Illke Evrim SECINTI (1) : 0000-0002-8614-3971

Altug KUCUKGUL : 0000-0001-6791-1595 
Anticarcinogenic, antihypertensive, immunomodulatory, anti-inflammatory, strong antioxidant, antiallergenic, neuroprotective, antimicrobial, hypolipidemic, vasodilator, antihypercholesterolemic, UV and radiation protective, hepatoprotective, estrogenic, analgesic, and sedative effects of hesperidin a flavonoid have been reported in pharmacological studies $(3,4,8,23,30,31,37)$. Hajialyani et al. reported that hesperidin effectively protected neurons from damage caused by oxidative or nitrosative stress (13).

Anti-inflammatory and antioxidant effects of hesperidin in mechanical, toxic, and ischemic injuries occurring in various tissues have been demonstrated in several studies; however, no study has assessed its effectiveness following acute spinal cord injury. Although there are several studies on hesperidin, the presente study is the first to evaluate its effectiveness against secondary injury after acute ( 24 hours) spinal cord trauma. The aim of the present study was to investigate the effectiveness of hesperidin in preventing secondary injury after experimental acute spinal cord trauma using clinical, biochemical, immunohistochemical, and histopathological analyses.

\section{- MATERIAL and METHODS}

This in vivo trial was approved by Hatay Mustafa Kemal University Animal Experiments Local Ethics Committee (dated 31.10.2016 and numbered 2016/9-7).

\section{Animals}

Rats were housed in the laboratory under optimal conditions and a 12-hour (h) day/night cycle, with species-specific pellet feed and water provided ad libitum. A total of 32 Wistar albino male rats weighing 250-300 g were used. The experimental animals were randomly divided into four groups as follows: group I, control group; group II, sham group: the group in which only spinal cord trauma was induced; group III, preconditioning group: the group that was administered 200 $\mathrm{mg} / \mathrm{kg} /$ day oral (po) hesperidin for 7 days before trauma; and group IV, treatment group: the group that was administered $200 \mathrm{mg} / \mathrm{kg}$ po hesperidin after trauma.

\section{Intervention}

Hesperidin (Sigma, USA) was dissolved in drinking water and administered by gavage. The animals were housed in individual cages and observed. Under anesthesia, intracardiac blood was collected for analyses from the control group and 24 hours after trauma from the other groups, and tissue samples were obtained from the trauma region of the spinal cord after the animals were sacrificed.

\section{Inducing Spinal Cord Injury}

For general anesthesia, $10 \mathrm{mg} / \mathrm{kg}$ of xylazine hydrochloride (intraperitoneal) (Alfazyne $2 \%$ injection $50 \mathrm{~mL}$, EGE-VET, Turkey) and $50 \mathrm{mg} / \mathrm{kg}$ ketamine hydrochloride (intrap) (Alfamine $10 \%$ injection $50 \mathrm{~mL}$ EGE-VET, Turkey) were administered to rats in all groups undergoing surgical intervention. After the anesthetized rats were fixed in the sternal position, the back region was broadly shaved and antisepsis was provided with povidone iodine. Paravertebral muscles were reached after passing the cutaneous and subcutaneous tissues with a 2-cm incision at T5-T12 level with reference to the interscapular distance (Figure 1). Paravertebral muscles were dissected and processus spinosi and vertebral laminae were reached. At the T7-T10 level, the spinal cord was exposed with total laminectomy without disrupting the integrity of the dura mater (Figure 2). Spinal cord injury was induced by the weight dropping method using a $10-\mathrm{g} 3-\mathrm{mm}$ diameter metal rod dropped from a height of $10 \mathrm{~cm}(100 \mathrm{~g} / \mathrm{cm})$. This induced paraplegia. Following hemostasis, paravertebral muscles and skin were sutured in accordance with their anatomical folds.

\section{Neurological Examination}

Inclined plane test: The inclined plane test described by Rivliv and Tator was used for experimental spinal cord injuries (38).

Clinical motor examination: Modified Tarlov scale (MTS) was used for motor examination (10) (Table I).

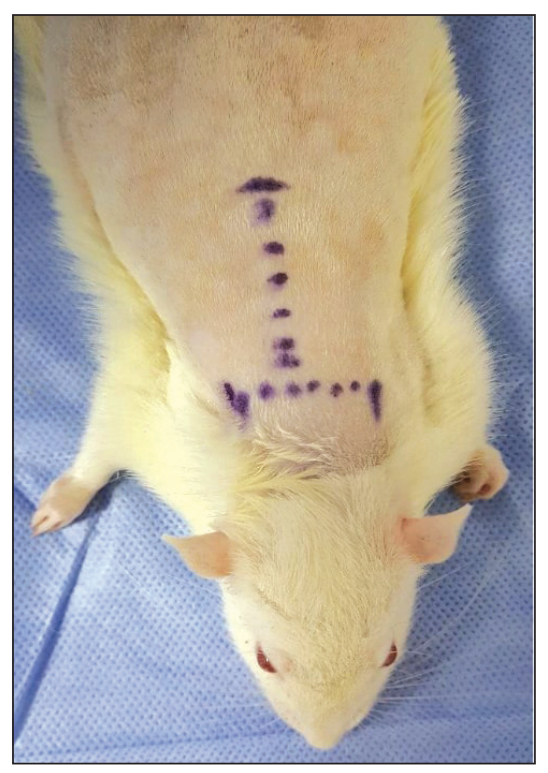

Figure 1: Determination of the interscapular distance.

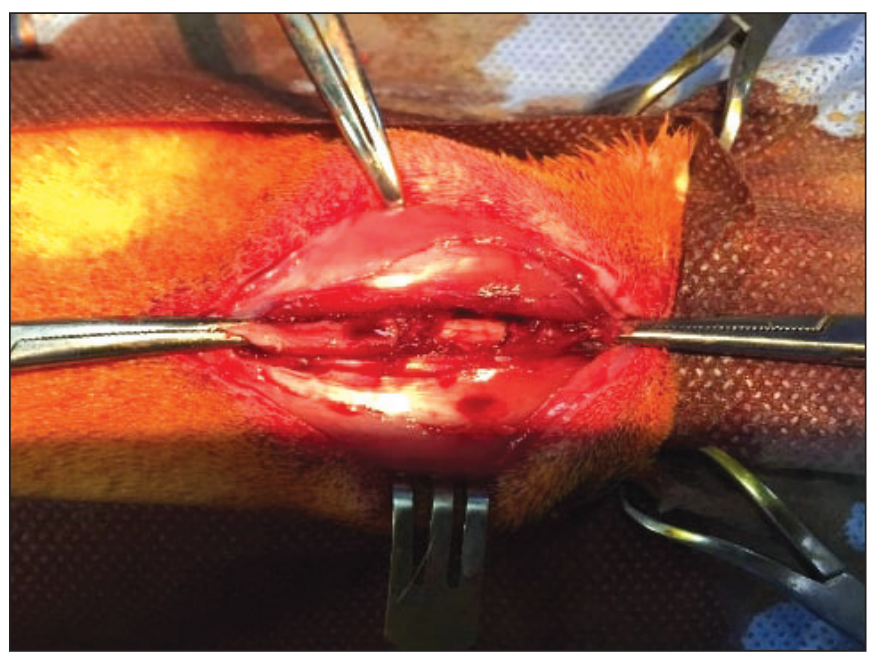

Figure 2: Exposing the spinal cord with dorsal laminectomy. 
Table I: Modified Tarlov Scale Score (10)

\begin{tabular}{cl}
\hline Degree & Modified Tarlov Scale \\
\hline 0 & Complete paralysis in the back extremities, no movement in the back extremities, no weight bearing \\
\hline 1 & Noticeable back limb movements, no weight bearing \\
\hline 2 & $\begin{array}{l}\text { Frequent and / or strong back limb movement, pronounced posterior limb movements that do not result in weight } \\
\text { overlay or locomotion }\end{array}$ \\
\hline 3 & Back extremities support body weight, can take one or two steps \\
\hline 5 & There is a slight loss in walking \\
\hline
\end{tabular}

Finger extension test: Rats were lifted from the plane, and hind limbs were suspended. Extension of the fingers was observed, and the reflexes were classified as follows (10): 0 , no extension; 1, moderate extension; and 2, full extension.

\section{Histopathological Evaluation}

In the spinal cord samples obtained after the trial, hemorrhage, laceration, necrosis, edema, neuronal and axonal degeneration, and inflammatory cell infiltration parameters were scored by routine histopathological method (H\&E staining) as described by Öztürk et al. (29). In this method, the score ranges between 0 and 3 ( $0=$ none, $1=$ mild, $2=$ moderate, $3=$ severe $)$.

\section{Immunohistochemical Analysis}

For immunohistochemical applications, 4-5- $\mu$ m-thick sections were obtained from tissue paraffin blocks using a rotary microtome. The prepared sections were stained with caspase 3 antibodies (1:1000 dilution; anti-caspase-3 [EPR18297] (ab184787), ABCAM, Cambridge, UK) using the combined streptavidin-biotin-peroxidase and microwave antigen retrieval method.

Caspase 3-positive cells were manually counted under a highpower field (400x), and the average number of positive cells was calculated in six different non-overlapping fields of vision (three gray matter and three white matter fields) on each slide (24). The preparations selected to represent each group were photographed under an Olympus BX 53 microscope using an Olympus DP2 BSW image analysis system.

\section{Biochemical Analyses}

The bioactivity of hesperidin under oxidative stress and inflammation was investigated in plasma samples obtained from experimental animals. Two oxidative stress parameters as well as total antioxidant and oxidant capacities were investigated via spectrophotometric analyses. Levels of two inflammatory parameters, namely interleukin 1-beta (IL$1 \beta$ ) and tumor necrosis factor alpha (TNF-a) protein, were investigated using enzyme-linked immunosorbent assay.

\section{Statistical Analysis}

Statistical analyses were performed using SPSS 17. Differences between the groups were analyzed using oneway ANOVA with Bonferroni test. Data are expressed as mean and standard error. A $\mathrm{p}<0.05$ was considered statistically significant.

\section{RESULTS}

\section{Neurological Findings}

Neurological examination using MTS revealed higher scores in the preconditioning group than in the sham and treatment groups(Table II). In the inclined plane test, significant differences were observed between the sham and treatment groups $(p<0.05$; Table II). Furthermore, in neurological examination using the finger extension test, significant differences were observed between the sham and preconditioning groups (p<0.05; Table II).

\section{Biochemical Findings}

The level of IL-1 $1 \beta$, a proinflammatory cytokine, was higher in the sham group $(107 \pm 7.0)$ than in the control group (84.33 \pm 3.97 ). Hesperidin preconditioning suppressed inflammation by lowering this $\mathrm{IL}-1 \beta$ level to the level of the control group $(85.16 \pm 1.53, p<0.01)$. In addition, IL-1 $\beta$ level was significantly decreased in the treatment group $(82.83 \pm 1.07, p<0.01)$. TNF-a level was higher in the sham group (593.66 \pm 80.7$)$ than in the control group $(446.0 \pm 59.8)$. However, post-traumatic hesperidin administration reduced TNF-a level in the treatment group (533.5 \pm 45.6 ; Table III).

Hesperidin showed a positive effect by significantly increasing TAS level in the preconditioning $(0.952 \pm 0.06, p<0.01)$ and treatment $(1.021 \pm 0.03, p<0.01)$ groups compared with that in the sham group (Table III). Hesperidin preconditioning decreased TOS level compared to sham (7.50 \pm 0.56 ); however, TOS level was slightly higher in the treatment group $(8.20 \pm 0.55$; Table III) than in the preconditioning group.

\section{Histopathological Findings}

Routine histopathological (H\&E staining) examination revealed normal findings in the control group (Figure 3A). In the sham group, necrotic area was scored 3 in histological sections. Considerable laceration, diffuse hemorrhage, intense edema, and neuronal degeneration (Nissl losses and red degeneration) were observed in the gray matter, and edema, intense axonal degeneration, and spherule structures were observed in the white matter (Figure 3B). Polymorphonuclear leukocyte 
Yurtal Z. et al: Effects of Hesperidin on Spinal Cord Injury

Table II: Effects of Hesperidin on Modified Tarlov Test in Experimental Spinal Cord Injury

\begin{tabular}{lccc}
\hline Groups & Modified Tarlov & Inclined Planed Test & Finger Opening Test \\
\hline Grup 1: Control & Mean \pm SE & Mean \pm SE & Mean \pm SE \\
\hline Grup 2: Sham & $5.00 \pm 0.0$ & $81.87 \pm 2.03$ & $2.00 \pm 0.00$ \\
\hline Grup 3: Preconditioning & $1.25 \pm 0.25 \neq$ & $69.50 \pm 2.12$ & $0.12 \pm 0.12 \neq$ \\
\hline Grup 4: Treatment & $2.00 \pm 0.50 \neq$ & $63.87 \pm 3.93 \beta$ & $0.50 \pm 0.32 \neq$ \\
\hline
\end{tabular}

In Modified Tarlov scale, the differences between the control and sham groups as well as between the preconditioning and treatment groups were significant $(p<0.001)$.

${ }^{\beta}$ In Inclined planed test, the differences between the control and preconditioning groups as well as between the preconditioning and treatment groups were significant $(p<0.005)$.

$\mu$ In the Inclined planed test, the differences between the sham and treatment group were significant $(p<0.05)$.

*In the finger extension test, the differences between the control and sham groups as well as between the preconditioning and treatment groups were significant $(p<0.001)$.

Table III: Effects of Hesperidin on IL-1ß, TNF-a, TAS, and TOS Levels in Experimental Spinal Cord Injury

\begin{tabular}{|c|c|c|c|c|c|}
\hline & $\mathbf{N}$ & Control & Sham & Preconditioning & Treatment \\
\hline & & Mean \pm SE & Mean \pm SE & Mean \pm SE & Mean \pm SE \\
\hline $\mathrm{IL}-1 \beta$ & 6 & $84.33 \pm 3.97$ & $107.0 \pm 7.03$ & $85.16 \pm 1.53 a$ & $82.83 \pm 1.07 \mathrm{a}$ \\
\hline TNF-a & 6 & $446.0 \pm 59.8$ & $593.66 \pm 80.7$ & $565.0 \pm 48.4$ & $533.5 \pm 45.6$ \\
\hline TAS & 6 & $0.650 \pm 0.08$ & $0.661 \pm 0.03$ & $0.952 \pm 0.06 a$ & $1.021 \pm 0.03 a$ \\
\hline TOS & 6 & $7.26 \pm 0.26$ & $7.70 \pm 0.50$ & $7.50 \pm 0.56$ & $8.20 \pm 0.55$ \\
\hline
\end{tabular}

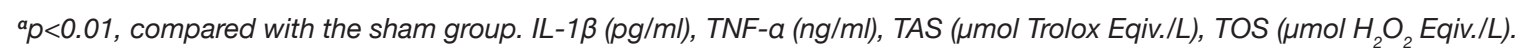
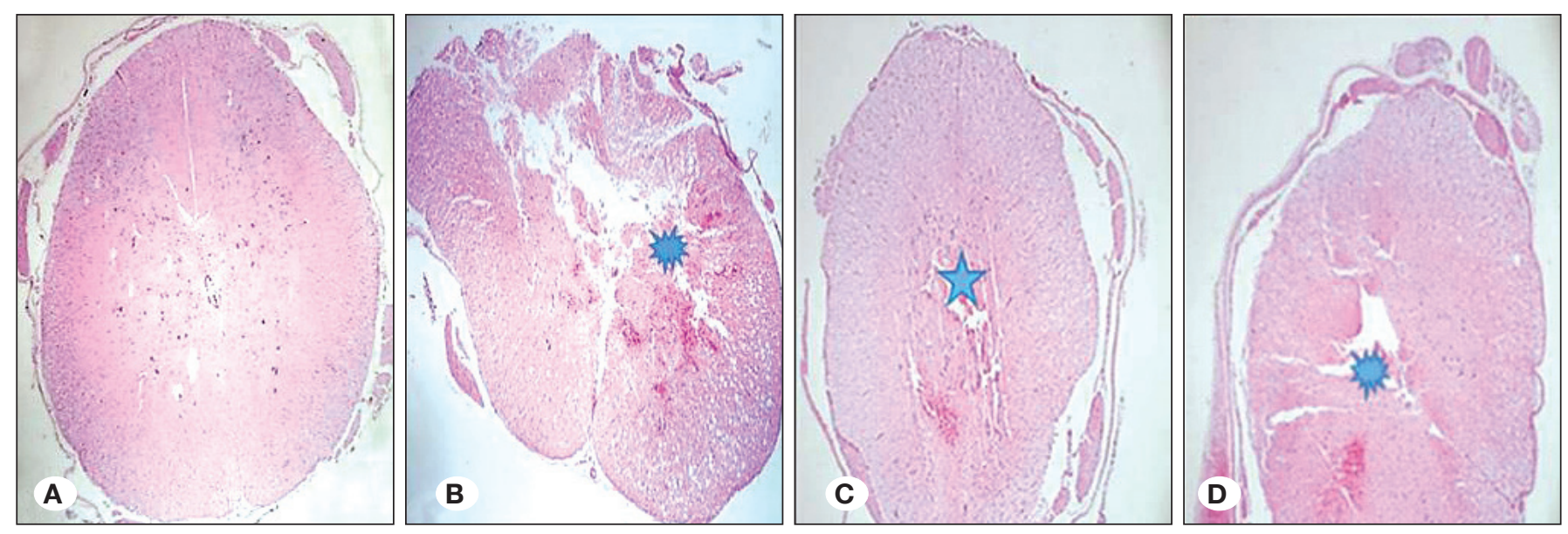

Figure 3: Histopathological images; A) control group, B) sham group, C) preconditioning group, D) treatment group.

(PMNL) infiltration was observed locally and was consistent with the typical findings observed during the acute period. Laceration, hemorrhage, edema, necrosis, axonal and neuronal degeneration, and neutrophil infiltration were significantly lower in the preconditioning group than in the sham group $(p<0.05$ to $p<0.001$; Figure $3 C$, Table IV). Hemorrhage, edema, necrosis, axonal and neuronal degeneration, and neutrophil infiltration were significantly lower in the treatment group than in the sham group $(p<0.05$ to $p<0.005$; Figure 3D and Table IV).

\section{Immunohistochemical Findings}

The numbers of caspase 3-positive apoptotic cells per unit area $(400 \times)$ are shown in Table V, and stained apoptotic cells are illustrated in Figure 4B-D. 
Table IV: Statistics of the Data in Histopathological Findings

\begin{tabular}{lcccc}
\hline & Control & Sham & Preconditioning & Treatment \\
\hline Hemorrhage & Meant \pm SE & Mean \pm SE & Mean \pm SE & Mean \pm SE \\
\hline Laceration & $0.125 \pm 0.12$ & $2.857 \pm 0.142$ & $1.833 \pm 0.307^{\beta}$ & $1.857 \pm 0.260^{\beta}$ \\
\hline Axonal and Neuronal Degeneration & $0.000 \pm 0.00$ & $2.857 \pm 0.142$ & $1.833 \pm 0.307^{*}$ & $1.857 \pm 0.260^{*}$ \\
\hline Necrosis & $0.000 \pm 0.00$ & $2.857 \pm 0.142$ & $1.333 \pm 0.494^{*}$ & $2.000 \pm 0.218$ \\
\hline Inflammatory Reaction & $0.000 \pm 0.00$ & $2.857 \pm 0.142$ & $1.666 \pm 0.333^{\beta}$ & $1.714 \pm 0.285^{\beta}$ \\
\hline Edema & $0.000 \pm 0.00$ & $2.714 \pm 0.184$ & $1.666 \pm 0.210^{\alpha}$ & $1.714 \pm 0.285^{a}$ \\
\hline
\end{tabular}

${ }^{*} p<0.05,{ }^{a} p<0.01,{ }^{\beta} p<0.005,{ }^{\neq} p<0.001$, compared with the sham group.

Table V: Statistical Data of Caspase-3 Immunireactivity

\begin{tabular}{ccccc}
\hline & Control & Sham & Preconditioning & Treatment \\
\hline & Mean \pm SE & Mean \pm SE & Mean \pm SE & Mean \pm SE \\
\hline Caspase 3 & $4.500 \pm 0.327$ & $28.71 \pm 2.168$ & $14.50 \pm 2.012^{\neq}$ & $12.42 \pm 1.797^{\neq}$ \\
\hline
\end{tabular}

${ }^{\neq} p<0.001$ compared with the control and sham group.

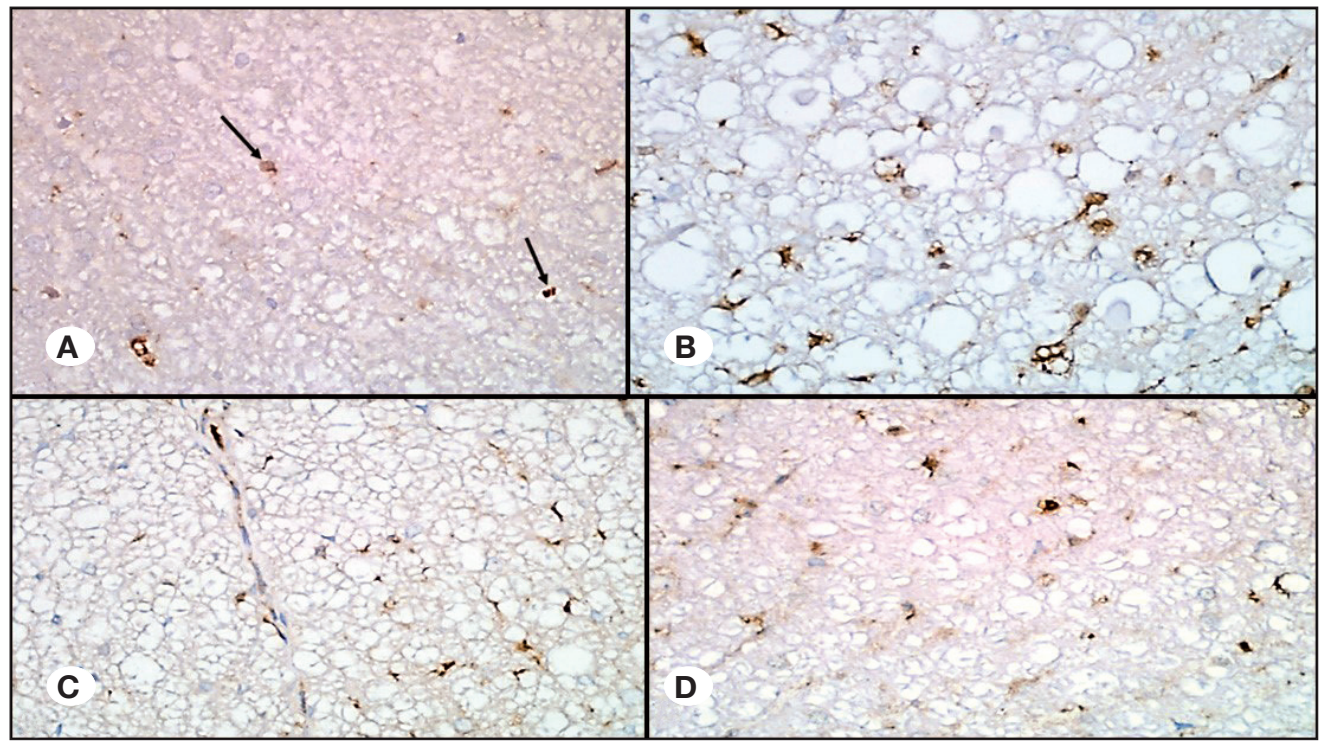

Figure 4: Caspase-3 images: A) Control group, B) Sham group, C) Preconditioning group, D) Treatment group.
Caspase 3 immunoreactivity was significantly lower in the control, preconditioning, and treatment groups than in the sham group $(p<0.001$; Figure $4 A-D$, Table V).

\section{DISCUSSION}

Although spinal cord injuries are considered an incurable condition (21), even a slight improvement in the motor or sensory functions considerably affects the patient's quality of life (33). Experimental spinal cord contusion models are frequently used to understand the pathophysiology of spinal cord injuries (40). Sengul et al. emphasized that it is impossible to prevent primary injury; therefore, treatment strategies against spinal cord injuries are aimed at preventing secondary injuries (36). Contusion models are important tools for exploring progressive secondary tissue injury, demyelination, and apoptosis in spinal cord injury (40). In the present study, the efficacy of hesperidin in preventing secondary injuries after spinal cord injury and its clinical benefits were investigated.

Several studies have reported that antioxidants can delay the progression of neurodegeneration (3). Zhang et al. applied compression on the spinal cord at the T8 level in rats and demonstrated locomotor improvement in MTS scores with combined anti-inflammatory therapy (41). In the present study, MTS scores in the preconditioning group were higher 
than those in the other groups, suggesting that hesperidin contributed to clinical recovery following trauma (Table II). Onifer et al. reported an inclined plane angle of $80^{\circ}$ among healthy animals in the inclined plane test (27). In the present study, the values obtained in the inclined plane test were higher in the preconditioning group than in the treatment group. Thus, hesperidin preconditioning provides clinical benefits (Table II). Finger extension scores decreased following trauma; however, an increase in these scores was observed in hesperidin-treated groups (Table II). Similar to the findings of the present study, Sirin et al. reported a considerable decrease in finger extension test scores following spinal cord injury (38).

According to the findings of the present study, hesperidin showed a positive effect by remarkably increasing TAS level in the preconditioning and treatment groups (Table III). In addition, hesperidin remarkably contributed to improvement by reducing TOS level in 24 hours. Consistent with our results, Tamilselvam et al. investigated the effect of hesperidin in an in vitro neurodegeneration model and reported that hesperidin suppressed the production of reactive oxygen radicals (which are among total oxidant molecules); increased the activity of glutathione peroxidase (GPx), superoxide dismutase (SOD), and catalase (CAT) (which are members of the total antioxidant system); and showed antioxidant activity by remarkably increasing the level of glutathione (GSH) (which is an intracellular antioxidant molecule) (39). Similarly, Liu et al. showed that in the rat model of diabetic retinopathy, hesperidin showed a positive effect on TAS, specifically by increasing SOD, CAT, and GSH levels (25). In addition, Selmi et al. reported that hesperidin showed antioxidant effects by remarkably reducing hydrogen peroxide and malondialdehyde levels, which are involved in oxidative stress (35). In the same study, they reported that hesperidin considerably increased SOD, CAT, and Gpx enzyme activities, which are the three parameters of antioxidant capacity (35). Thus, the mechanism of action of hesperidin in recovery during the acute period of spinal cord injury is via increase in TAS levels and decrease in TOS levels. Furthermore, the hesperidin-mediated increase in TOS levels in the treatment group triggered acute inflammation, indicating chemotactic activity, particularly for tissue repairing cells.

In the present study, hesperidin reduced inflammation by decreasing IL-1 $\beta$ levels in the preconditioning and treatment groups compared with those in the sham group. In addition, hesperidin reduced inflammation and showed therapeutic efficacy by decreasing TNF-a levels in the preconditioning and treatment groups compared with those in the sham group. Consistent with these results, Selmi et al. reported that hesperidin remarkably suppressed increased TNF-a expression and showed anti-inflammatory activity (35). Cheng et al. reported that citrus flavonoids such as hesperidin show strong anti-inflammatory activity by reducing TNF-a, IL-1 and IL-6 levels (6). Consistent with our results, Ansar et al. reported that hesperidin shows anti-inflammatory activity in nanoparticle-induced neurotoxicity by suppressing TNF-a and $\mathrm{IL}-1 \beta$ gene expression (2). One of the mechanisms underlying the impact of hesperidin on recovery observed in the present study might be its suppressive effects on inflammatory gene expression.
Heo et al. reported that hesperidin showed anti-inflammatory and antioxidant effects in the subacute period (4 days) in their spinal cord injury model (15). Similar to these findings, hesperidin showed anti-inflammatory and antioxidant properties in the acute period (24 h) in spinal cord injury model in the present study.

Sengul et al. reported that histopathological evaluation in the physiological saline group showed a considerable increase in hemorrhage, edema, neuronal degeneration, and inflammation in a spinal cord injury model (36). In the present study, considerable laceration, diffuse hemorrhage, intense edema, and neuronal degeneration (Nissl losses and red degeneration) were observed in the gray matter and edema, intense axonal degeneration, and spherule structures were observed in the white matter in the sham group (Figure 3B). PMNL infiltration was observed locally and was consistent with the typical findings observed during the acute period. Hesperidin improved all these findings. Ahuja et al. reported that secondary spinal cord injury begins following primary spinal cord injury, and inflammatory cells such as macrophages, microglia, T-cells, and neutrophils infiltrate into the injured area as a result of disruption of the blood-spinal cord barrier (1). Kjell and Olson stated that neutrophils infiltrate the injury site during the first 3-6 h after spinal cord injury, monocyte infiltration begins on the 3rd day after injury, and neutrophils are probably no longer observed in the area due to apoptosis (22). Moreover, Ahuja et al. reported that during the post-traumatic acute injury period $(0-48 \mathrm{~h})$, hemorrhage, edema, and proapoptotic factors (cytokines, $\mathrm{K}^{+}, \mathrm{DNA}$, and necrotic tissue, among others) contribute to ongoing cell death (1). Consistent with previous studies, hemorrhage, edema, laceration, and neuronal degeneration were noted following acute injury in the present study.

Lin et al. stated that various molecular pathways such as hypoxia, ischemia, lipid peroxidation, free radical production, neutral protease activation, prostaglandin production, and programmed cell death or apoptosis may be involved in the underlying pathologies of spinal cord injury (24). They also stated that the primary injury in the spinal cord disrupts the cell membranes, destroys the myelin and axons, and triggers secondary injury by disrupting microcirculation, resulting in a destructive effect (24). They emphasized that secondary injury cascades, including local inflammation, free radical production, and hyperoxidation, are active biological processes, and as a result, some neurons and glial cells die even if patients recover from the injury that occurs days or weeks after spinal cord injury (24). Gaur and Kumar stated that in a rat model of bilateral carotid ischemia-reperfusion, hesperidin pretreatment for 7 days improved memory recovery and showed protective effects on cerebral ischemia histopathologically (11). Zhang et al. reported that acute inflammatory response in spinal cord injuries is an initial reaction that leads to endothelial cell damage and vascular integrity loss and is subsequently characterized by a stage at which neutrophil activities predominate and necrosis and cavitation occur during the later period (41). Norenberg et al. stated that after acute injuries following spinal cord damage, spheroid structures with hemorrhage and axonal 
swelling were formed, and hemorrhage occurred due to the rupture of postcapillary venules and sulcal arterioles in the gray matter (26). Consistent with these reports, widespread hemorrhage and neutrophil infiltration were observed during the acute period in the present study. In addition, axonal degeneration and spherule structures along with edema were observed in the white matter in the sham group (Figure 3C). Laceration, hemorrhage, edema, necrosis, axonal and neuronal degeneration, and neutrophil leukocyte infiltration decreased in the preconditioning group compared with those in the sham group. Moreover, hemorrhage, laceration, edema, axonal and neuronal degeneration slightly decreased in the treatment group compared with those in the sham group. Thus, histopathologically, hesperidin use showed therapeutic efficacy in both the preconditioning and treatment groups by decreasing all parameters such as hemorrhage, laceration, axonal and neuronal degeneration, necrosis, inflammatory reaction, and edema.

Oztanir et al. clamped the carotid artery for 15 minutes in a mouse model of ischemia-reperfusion (IR) and found that the number of caspase 3-positive cells remarkably increased in the IR group (28). They stated that the proportion of immunohistochemical caspase 3-positive cells decreased in the IR + hesperidin group compared with that in the IR group; therefore, hesperidin treatment considerably reduced the IRinduced increase in the number of caspase 3-positive cells (28). Roohbakhsh et al. stated that the neuroprotective effect of hesperidin mainly occurred via its antioxidant and antiinflammatory activities and attributed weakened caspase-3 activity, calcium ion regulation, improved neuronal energy metabolism, and reversed mitochondrial dysfunction to the neuroprotective effects of hesperidin (32).

Kamisli et al. demonstrated the antiapoptotic activity of hesperidin and showed that hesperidin reduced neuronal degeneration caused by cisplatin (19). Hwang and Yen created an in vitro cytotoxicity model of $\mathrm{PC} 12$ cells induced with $\mathrm{H}_{2} \mathrm{O}_{2}$ and reported that hesperidin, hesperetin, and neohesperidin weakened the increase in caspase- 3 activity and showed neuroprotective activities (17). Ciftci et al. investigated the healing effects of hesperidin in a mouse model of experimental autoimmune encephalomyelitis (EAE) and, based on their histopathological and immunohistochemical (caspase-3) evaluation, reported that histological damage and apoptosis considerably increased in the EAE group and considerably decreased in the hesperidin group compared with those in the other groups (7). Tamilselvam et al. reported that hesperidin showed a neuroprotective effect by activating signaling pathways such as Akt and ERK $1 / 2$ and preventing the release of proapoptotic proteins such as apoptosis signal regulating kinase 1, BAD, and caspase 3 and 9 (39). In the present study, hesperidin decreased the number of cells showing caspase 3 immunoreactivity in the preconditioning and treatment groups compared with that in the sham group $(p<0.001)$. These results indicate that hesperidin reduces apoptosis by affecting the caspase 3 pathway and shows a neuroprotective effect.

\section{- CONCLUSION}

Hesperidin shows antioxidant, anti-inflammatory, and antiapoptotic effects during the acute period after spinal cord injury and exhibits neuroprotective and therapeutic efficacy. At the tissue level, these effects reflect positively on neurological findings.

\section{ACKNOWLEDGMENTS}

This study was financially supported by the Scientific Research Projects Coordination Unit of Hatay Mustafa Kemal University (Project No. 16522).

\section{REFERENCES}

1. Ahuja CS, Nori S, Tetreault L, Wilson J, Kwon B, Harrop J, Choi D, Fehlings MG: Traumatic spinal cord injury repair and regeneration. Neurosurgery 80(3S):S9-S22, 2017

2. Ansar S, Abudawood M, Hamed SS, Aleem MM: Exposure to zinc oxide nanoparticles induces neurotoxicity and proinflammatory response: Amelioration by hesperidin. Biological Trace Element Research 175(2):360-366, 2017

3. Banji OJF, Banji D, Ch K: Curcumin and hesperidin improve cognition by suppressing mitochondrial dysfunction and apoptosis induced by D-galactose in rat brain. Food Chem Toxicol 74:51-59, 2014

4. Carballo-Villalobos AI, González-Trujano ME, Pellicer F, LópezMuñoz FJ: Antihyperalgesic effect of hesperidin improves with diosmin in experimental neuropathic pain. BioMed Research International 2016:8263463, 2016

5. Celik H, Mut S, Harman F, Yilmaz G, Berkman MZ: Deneysel spinal kord yaralanmasında vitamin D3'ün motor fonksiyonlar üzerine olan iyileştirici etkisi. Turk J Neurol 21(2):55-61, 2015

6. Cheng L, Ren Y, Lin D, Peng S, Zhong B, Ma Z: The antiinflammatory properties of citrus wilsonii tanaka extract in LPS-induced RAW 264.7 and primary mouse bone marrowderived dendritic cells. Molecules 22(7):1213, 2017

7. Ciftci O, Ozcan C, Kamisli O, Cetin A, Basak N, Aytac B: Hesperidin, a citrus flavonoid, has the ameliorative effects against experimental autoimmune encephalomyelitis (EAE) in a C57BL/J6 mouse model. Neurochemical Research 40(6):1111-1120, 2015

8. Cimen MBY: Flavonoidler ve antioksidan özellikleri. T Klin Tıp Bilimleri 19:296-304, 1999

9. Cristante AF, Barros Filho TEP, Marcon RM, Letaif OB, Rocha ID: Therapeutic approaches for spinal cord injury. Clinics 67(10):1219-1224, 2012

10. Gale K, Kerasidis H, Wrathall JR: Spinal cord contusion in the rat: Behavioral analysis of functional neurologic impairment. Experimental Neurology 88(1):123-134, 1985

11. Gaur V, Kumar A: Hesperidin pre-treatment attenuates NO mediated cerebral ischemic reperfusion injury and memory dysfunction. Pharmacol Rep 62(4):635-648, 2010

12. Gurcan O, Gurcay AG, Kazanci A, Senturk S, Bodur E, Karaca EU, Turkoglu OF, Bavbek M: Effect of asiatic acid on the treatment of spinal cord injury: An experimental study in rats. Turkish Neurosurgery 27(2):259-264, 2017 
Yurtal Z. et al: Effects of Hesperidin on Spinal Cord Injury

13. Hajialyani M, Hosein Farzaei MH, Echeverría J, Nabavi SM, Uriarte E, Sobarzo-Sánchez E: Hesperidin as a neuroprotective agent: $A$ review of animal and clinical evidence. Molecules 24(3):648, 2019

14. Hanci V, Kerimoglu A, Koca K, Baskesen A, Kilic K, Tastekin $D$ : The biochemical effectiveness of $\mathrm{N}$-acetylcysteine in experimental spinal cord injury in rats. Ulus Travma Acil Cerrahi Derg 16(1):15-21, 2010

15. Heo SD, Kim J, Choi Y, Ekanayaki P, Ahn M, Shin T: Hesperidin improves motor disability in rat spinal cord injury through antiinflammatory and antioxidant mechanism via $\mathrm{Nrf}-2 / \mathrm{HO}-1$ pathway. Neoroscience Letter 715:134619, 2019

16. Hou QX, Yu L, Tian SQ, Jiang CJ, Yang WJ, Wang ZJ: Neuroprotective effects of atomoxetine against traumatic spinal cord injury in rats. Iranian Journal of Basic Medical Sciences 19(3):272-280, 2016

17. Hwang SL, Yen GC: Neuroprotective effects of the citrus flavanones against $\mathrm{H} 2 \mathrm{O} 2$-induced cytotoxicity in $\mathrm{PC} 12$ cells. Journal of Agricultural and Food Chemistry 56(3):859-864, 2008

18. Istan GK, Erbayraktar Z, Gokmen N, Bagriyanik A, Kiray M, Erbayraktar SR: Deneysel omurilik yaralanmasında gabapentinin nöroprotektif etkinliğinin değerlendirilmesi. Sinir Sistemi Cerrahisi Derg 4(4):169-184, 2014

19. Kamisli S, Ciftci O, Kaya K, Cetin A, Kamisli O, Ozcan C: Hesperidin protects brain and sciatic nerve tissues against cisplatin-induced oxidative, histological and electromyographical side effects in rats. Toxicology and Industrial Health 31(9):841-851, 2015

20. Kermani HR, Nakhaee N, Fatahian R, Najar AG: Effect of aspirin on spinal cord injury: An experimental study. Iranian Journal of Medical Sciences 41(3):217-222, 2016

21. Kim YH, Ha KY, Kim SI: Spinal cord injury and related clinical trials. Clinics in Orthopedic Surgery 9(1):1-9, 2017

22. Kjell J, Olson L: Rat models of spinal cord injury: From pathology to potential therapies. Disease Models and Mechanisms 9(10):1125-1137, 2016

23. Kuntić V, Brborić J, Holclajtner-Antunović I, UskokovićMarković S: Evaluating the bioactive effects of flavonoid hesperidin-A new literature data survey. Vojnosanitetski Pregled 71(1):60-65, 2014

24. Lin MS, Lee YH, Chiu WT, Hung KS: Curcumin provides neuroprotection after spinal cord injury. The Journal of Surgical Research 166(2):280-289, 2011

25. Liu WY, Liou SS, Hong TY, Liu IM: Protective effects of hesperidin (Citrus Flavonone) on high glucose induced oxidative stressand apoptosis in a cellular model for diabetic retinopathy. Nutrients 9(12):1312, 2017

26. Norenberg MD, Smith J, Marcillo A: The pathology of human spinal cord injury: Defining the problems. Journal of Neurotrauma 21(4):429-440, 2004

27. Onifer SM, Rabchevsky AG, Scheff SW: Rat models of traumatic spinal cord injury to assess motor recovery. ILAR Journal 48(4):385-395, 2007
28. Oztanir MN, Ciftci O, Cetin A, Aladag MA: Hesperidin oxidative and neuronal damage caused by global cerebral ischemia/reperfusion in a C57BL/J6 mouse model. Neurol Sci 35(9):1393-1399, 2014

29. Öztürk ÖÇ, Gürer B, Kertmen H, Bozkurt H, Kasım E, Kanat MA, Arıkök AT, Ergüder Bi, Sargon MF, Şekerci Z: Progesteron'un tavşan spinal kord iskemi/reperfüzyon hasarında nöroprotektif etkileri. Türk Nöroşir Derg 26(3):207-217, 2016

30. Polat N, Ciftci O, Cetin A, Yılmaz T: Toxic effects of systemic cisplatin on rat eyes and the protective effect of hesperidin against this toxicity. Cutaneous and Ocular Toxicology 35(1):1 7, 2016

31. Rezaeyan A, Haddadi GH, Hosseinzadeh M, Moradi M, Najafi M: Radioprotective effects of hesperidin on oxidative damages and histopathological changes induced by $X$-irradiation in rats heart tissue. Journal of Medical Physics 41(3):182-191, 2016

32. Roohbakhsh A, Parhiz H, Soltani F, Rezaee R, Iranshahi M: Neuropharmacological properties and pharmacokinetics of the citrus flavonoids hesperidin and hesperetin-A mini-review. Life Sciences 113(1-2):1-6, 2014

33. Rouanet C, Reges D, Rocha E, Gagliardi V, Silva GS: Traumatic spinal cord injury: Current concepts and treatment update. Arquivos de Neuro-Psiquiatria 75(6):387-393, 2017

34. Sahin Kavakli H, Koca C, Alici O: Antioxidant effects of curcumin in spinal cord injury in rats. Turkish Journal of Trauma and Emergency Surgery 17(1):14-18, 2011

35. Selmi S, Rtibi K, Grami D, Sebai H, Marzouki L: Protective effects of orange (Citrus sinensis L.) peel aqueous extract and hesperidin on oxidative stress and peptic ulcer induced by alcohol in rat. Lipids in Health and Disease 16(1):152, 2017

36. Sengul G, Coban MK, Cakır M, Coskun S, Aksoy H, Hacımuftuoglu A, Saruhan F, Calık M: Neuroprotective effect of acute interferon-beta $1 \mathrm{~b}$ treatment after spinal cord injury. Turkish Neurosurgery 23(1):45-49, 2013

37. Subramanian $\mathrm{P}$, Anandan R, Jayapalan JJ, Hashim $\mathrm{OH}$ : Hesperidin protects gentamicin-induced nephrotoxicity via $\mathrm{Nrf} / \mathrm{HO}-1$ signaling and inhibits inflammation mediated by NF-KB in rats. Journal of Functional Foods 13:89-99, 2015

38. Sirin YS, Keles H, Besaltı O, Atalay Vural S: Comparison of ATP-MgCl2 and methylprednisolone in experimental induced spinal cord trauma. Journal of Clinical and Analytical Medicine 3(4):442-447, 2012

39. Tamilselvam K, Braidy N, Manivasagam T, Essa MM, Prasad NR, Karthikeyan S, Thenmozhi AJ, Selvaraju S, Guillemin GJ: Neuroprotective effects of hesperidin, a plant flavanone, on rotenone-induced oxidative stress and apoptosis in a cellular model for Parkinson's disease. Oxidative Medicine and Cellular Longevity 8:1-11, 2013

40. Young W: Spinal cord contusion models. Progress in Brain Research 137:231-255, 2002

41. Zhang Z, Krebs CJ, Guth L: Experimental analysis of progressive necrosis after spinal cord trauma in the rat: Etiological role of the inflammatory response. Experimental Neurology 143(1):141-152, 1997 